\title{
Hardy type inequalities for superquadratic functions via Jackson Nörlund integrals
}

\author{
Hafiz Abdul Moeed ${ }^{a}$, Dawood Ahmad ${ }^{a}$, Ammara Nosheen $^{a}, *$, Khuram Ali Khan ${ }^{b}$ \\ ${ }^{a}$ Department of Mathematics, University of Lahore, Sargodha Campus, Pakistan \\ ${ }^{b}$ Department of Mathematics, University of Sargodha, Sargodha, Pakistan.
}

\begin{abstract}
In this paper, it is tried to describe Hardy-type inequalities with certain kernels by using Jackson Nörlund integrals. In order to obtain the desired Hardy type inequalities, firstly, we prove Jensen's inequality involving super quadratic function and Jackson Nörlund integrals. Further, we discuss Hardy-type inequalities by choosing special kernels. Polya-Knopp type inequalities are also deduced to find applications.
\end{abstract}

Keywords: Hahn integral operators, superquadratic function, Hardy-type inequalities.

2020 MSC: 26D10, 26D15, 39B62, 26A51.

(C)2021 All rights reserved.

\section{Introduction}

\subsection{Hahn operators}

The Hahn differential operator (introduced in 1949), which is a beginner guitar for building orthogonal polynomial families and researching some issues of approximation, unifies (in the limit) the two most well-known and used Jackson q-difference operators namely the Jackson q-difference derivative $D_{q}$, for $\mathrm{q} \in] 0,1\left[\right.$ given in [5] and the forward difference $\Delta_{w}$, where $w>0$. In [9] Hahn difference operator $\mathrm{D}_{\mathrm{q}, \omega}$ has been introduced in the following way:

$$
\left.\mathrm{D}_{\mathrm{q}, \omega} \zeta(\mathrm{t}):=\frac{\zeta(\mathrm{q} t+\omega)-\zeta(\mathrm{t})}{\mathrm{t}(\mathrm{q}-1)+\omega}, \mathrm{t} \neq \frac{\omega}{1-\mathrm{q}}, \quad \mathrm{q} \in\right] 0,1[\omega>0
$$

It can be observed:

$$
\begin{aligned}
& \mathrm{D}_{\mathrm{q}, \omega} \zeta(\mathrm{t})=\Delta_{\omega} \zeta(\mathrm{t}), \quad \text { whenever } \mathrm{q}=1, \\
& \mathrm{D}_{\mathrm{q}, \omega} \zeta(\mathrm{t})=\mathrm{D}_{\mathrm{q}} \zeta(\mathrm{t}), \quad \text { whenever } \omega=0, \text { and } \\
& \mathrm{D}_{\mathrm{q}, \omega} \zeta(\mathrm{t})=\zeta^{\prime}(\mathrm{t}), \quad \text { whenever } \mathrm{q}=1, \omega \rightarrow 0 .
\end{aligned}
$$

\footnotetext{
${ }^{*}$ Corresponding author

Email addresses: moeedmaths@gmail.com (Hafiz Abdul Moeed), dawoodahmad.edu@gmail.com (Dawood Ahmad), hammaran@gmail.com (Ammara Nosheen), khuram.sms@gmail.com (Khuram Ali Khan)
}

doi: $10.22436 /$ jmcs.023.03.04

Received: 2020-03-31 Revised: 2020-04-17 Accepted: 2020-08-17 
However, in 2012 the construction of a proper inverse of $\mathrm{D}_{\mathrm{q}, w}$ or the associated integral (namely Jackson Nörlund integral) has been introduced by Aldwoah et al. [3]. [16] addresses the basic ideas of Hahn variational calculus, such as the equations of Euler Lagrange for isoperimetric problems, as well as optimal control issues. In [15] in order to generalize the Hahn calculus of differences, Malinowska and Martins had used Hahn variation calculus and got circumstances of transversality.

\subsection{Hardy inequalities}

"The discrete Hardy inequality asserts that if $p \leqslant 1$ and $\left\{A_{\varpi}\right\}_{1}^{\infty}$ is a sequence of nonnegative real number, then

$$
\sum_{n=1}^{\infty}\left(\frac{1}{n} \sum_{\varpi=1}^{n} A_{\varpi}\right)^{p} \leqslant\left(\frac{p}{p-1}\right)^{p} \sum_{n=1}^{\infty} a_{n}^{p}
$$

holds". The classical Hardy inequality states that if $g\left(z_{1}\right) \geqslant 0$ is integrable over any finite interval $\left(0, z_{1}\right)$ and $\zeta^{p}$ is integrable and convergent over $(0, \infty)$ and $p>1$, then for $G\left(z_{1}\right)=\int_{0}^{z_{1}} g(t) d t$, the following holds:

$$
\int_{0}^{\infty}\left(\frac{1}{z_{1}} \mathrm{G}\left(z_{1}\right)\right)^{\mathrm{p}} \leqslant\left(\frac{p}{1-p}\right)^{p} \int_{0}^{\infty} g^{p}\left(z_{1}\right) d z_{1} \quad p>1
$$

unless $\mathrm{g} \equiv 0$, the constant appeared in (1.1) is best possible. Hardy inequality has been generalized by Hardy himself. He showed that, "for any $m \neq 1, p>1$, and any integrable function $g\left(z_{1}\right) \geqslant 0$ on $(0, \infty)$, the following holds:

$$
\int_{0}^{\infty} z_{1}{ }^{-\mathrm{m}} \mathrm{G}^{\mathrm{p}}\left(z_{1}\right) \leqslant\left(\frac{\mathrm{p}}{|\mathrm{m}-1|}\right)^{\mathrm{p}} \int_{0}^{\infty} z_{1}^{-\mathrm{m}}\left[z_{1} \mathrm{~g}\left(z_{1}\right)\right]^{\mathrm{p}} \mathrm{d} z_{1}
$$

unless $g \equiv 0$, where the constant is also best possible". These inequalities can be found in [10]. Because of fundamental importance of Hardy type inequalities in the discipline, there has been a great deal of effort and time over the years to improve and generalize the inequalities of Hardy [7, 8, 11-14]. Whereas one of Hardy inequalities for superquadratic function, denoted by $\Upsilon$, proved in [2] is

$$
\Upsilon\left(\int_{\Omega} \zeta \mathrm{d} \mu\right) \leqslant \int_{\Omega}(\curlyvee \zeta(\mathrm{s}))-\curlyvee\left(\left|\zeta(\mathrm{s})-\int_{\Omega} f \mathrm{~d} \mu\right|\right) \mathrm{d} \mu \mathrm{s}
$$

for all non-negative probability measure $\mu$, and integrable function $\zeta$. However in time scales settings Hardy inequalities for superquadratic functions are established in [6].

Hardy type inequalities for convex functions by using Jackson Nörlund integrals can be seen in [4]. Now we extend the results of [4] for superquadratic function by using Jackson Nörlund integrals.

\section{Preliminaries}

\subsection{Superquadratic function}

A function $\Upsilon:[0, \infty) \rightarrow \mathbb{R}$ is said to be superquadratic [1] provided that for all $z_{1} \geqslant 0$ there exists a canstant $\mathrm{c}\left(z_{1}\right) \in \mathbb{R}$ such that

$$
\Upsilon\left(z_{2}\right)-\Upsilon\left(z_{1}\right)-\Upsilon\left(\left|z_{2}-z_{1}\right|\right) \geqslant c\left(z_{1}\right)\left(z_{2}-z_{1}\right)
$$

holds for all $z_{2} \geqslant 0$.

\subsection{Properties of superquadratic functions}

Let $\checkmark$ be a superquadratic function with $c\left(z_{1}\right) \in \mathbb{R}$, then

1. $\Upsilon(0) \leqslant 0$;

2. if $\Upsilon(0)=\Upsilon^{\prime}(0)=0$, then $c\left(z_{1}\right)=\Upsilon^{\prime}\left(z_{1}\right)$, whenever $\Upsilon$ is differentiable at $z_{1}>0$;

3. if $\Upsilon \leqslant 0$, then $\Upsilon$ is convex and $\Upsilon(0)=\Upsilon^{\prime}(0)=0$. 


\subsection{Jackson Nörlund integration}

“Let $I=\left[a, a^{\prime}\right]$ be a closed interval of $\mathbb{R}$. The $q, w$ integral [3] of $g_{1}: I \rightarrow \mathbb{R}$ is defined by

$$
\int_{a}^{a^{\prime}} g_{1}(t) d_{q, w}(t):=\int_{w_{0}}^{a^{\prime}} g_{1}(t) d_{q, w}(t)-\int_{w_{0}}^{a} g_{1}(t) d_{q, w}(t)
$$

and

$$
\int_{w_{0}}^{z_{1}} g_{1}(t) d_{q, w}(t)=\left(z_{1}(1-q)-w\right) \sum_{\varpi=0}^{\infty} q^{\varpi} g_{1}\left(z_{1} q_{\varpi}+w[\varpi]_{q, w}\right),
$$

where $w_{0}=\frac{w}{1-q},[\varpi]_{\mathbf{q}, w}=\frac{w\left(1-q^{\Phi}\right)}{1-q}$, and the series on the right hand side are convergent at $z_{1}=a$ and $z_{1}=a^{\prime \prime \prime}$.

\subsection{Properties of Jackson Nörlund integrals}

"Let $\mathrm{g}_{1}, \mathrm{~g}_{2}: \mathrm{I}_{1} \rightarrow \mathbb{R}$ be $\mathrm{q}, w$ integrable on $\mathrm{I}_{1}, \mathrm{c} \in \mathbb{R}$ and $\mathrm{a}, \mathrm{a}^{\prime}, \mathrm{b}, \mathrm{c} \in \mathrm{I}_{1}$, then

1. $\int_{a}^{a^{\prime}} g_{1}(t) d_{q, w} t=0$;

2. $\int_{a}^{a^{\prime}} c g_{1}(t) d_{q, w} t=c \int_{a}^{a^{\prime}} g_{1}(t) d_{q, w} t$;

3. $\int_{a}^{a^{\prime}} g_{1}(t) d_{q, w} t=-\int_{a^{\prime}}^{a} g_{1}(t) d_{q, w} t ;$

4. $\int_{a}^{a^{\prime}} g_{1}(t) d_{q, w} t=\int_{a}^{b} g_{1}(t) d_{q, w} t+\int_{b}^{a^{\prime}} g_{1}(t) d_{q, w} t ;$

5. $\int_{a}^{a^{\prime}} g_{1}(t) \pm g_{2}(t) d_{q, w} t=\int_{a}^{a^{\prime}} g_{1}(t) d_{q, w} t \pm \int_{a}^{a^{\prime}} g_{1}(t) d_{q, w} t . "$

\section{Main results}

\subsection{Jensen's inequality}

Theorem 3.1. Let $\mathrm{I}=\left[\mathrm{a}, \mathrm{a}^{\prime}\right] \subset \mathbb{R}$ be an interval. Assume that $\Upsilon \in \mathrm{C}(\mathrm{I}, \mathbb{R})$ is superquadratic. Moreover, $\zeta: \mathrm{I} \rightarrow \mathbb{R}$ is $\mathrm{q}, w$-integrable such that $\int_{\mathrm{a}}^{\mathrm{a}^{\prime}}|\zeta(\mathrm{l})| \mathrm{d}_{\mathrm{q}, w}(\mathrm{l})>0$, then the following holds:

$$
\int_{a}^{a^{\prime}}\left(\Upsilon(\zeta(l))-\Upsilon\left|\zeta(l)-\int_{a}^{a^{\prime}} \zeta(l) d_{q, w} l\right|\right) d_{q, w} l \geqslant \Upsilon\left(\int_{a}^{a^{\prime}} \zeta(l) d_{q, w} l\right) .
$$

Proof. Let

$$
z_{1}=\frac{1}{a^{\prime}-a} \int_{a}^{a^{\prime}} \zeta(l) d_{q, w} l
$$

By using (3.1) in (2.1) with $z_{2}=\zeta(l)$ for fixed $l \in I$, we have

$$
\begin{aligned}
\Upsilon(\zeta(l)) \geqslant & \Upsilon\left(\frac{1}{a^{\prime}-a} \int_{a}^{a^{\prime}} \zeta(l) d_{q, w} l\right)+c\left(z_{1}\right)\left(\zeta(l)-\frac{1}{a^{\prime}-a} \int_{a}^{a^{\prime}} \zeta(l) d_{q, w} l\right) \\
& +\Upsilon\left(\left|\zeta(l)-\frac{1}{a^{\prime}-a} \int_{a}^{a^{\prime}} \zeta(l) d_{q, w} l\right|\right) .
\end{aligned}
$$

Integrate (3.2) from $a$ to $a^{\prime}$ to get

$$
\begin{aligned}
& \int_{a}^{a^{\prime}}\left(\curlyvee(\zeta(l))-\Upsilon\left|\zeta(l)-\frac{1}{a^{\prime}-a} \int_{a}^{a^{\prime}} \zeta(l) d_{q, w} l\right|\right) d_{q, w} l-\int_{a}^{a^{\prime}} \Upsilon\left(\frac{1}{a^{\prime}-a} \int_{a}^{a^{\prime}} \zeta(l) d_{q, w} l\right) d_{q, w} l \\
& \quad=\int_{a}^{a^{\prime}} \curlyvee(\zeta(l)) d_{q, w} l-\int_{a}^{a^{\prime}} \Upsilon\left(\left|\zeta(l)-\frac{1}{a^{\prime}-a} \int_{a}^{a^{\prime}} \zeta(l) d_{q, w} l\right|\right) d_{q, w} l-\int_{a}^{a^{\prime}} \Upsilon\left(\frac{1}{a^{\prime}-a} \int_{a}^{a^{\prime}} \zeta(l) d_{q, w} l\right) d_{q, w} l
\end{aligned}
$$




$$
\begin{aligned}
& \geqslant c\left(z_{1}\right) \int_{a}^{a^{\prime}}\left(\left|\zeta(l)-\frac{1}{a^{\prime}-a} \int_{a}^{a^{\prime}} \zeta(l) d_{q, w} l\right|\right) d_{q, w} l \\
& =c\left(z_{1}\right)\left[\int_{a}^{a^{\prime}} \zeta(l) d_{q, w} l-\int_{a}^{a^{\prime}}\left(\frac{1}{a^{\prime}-a} \int_{a}^{a^{\prime}} \zeta(l) d_{q, w} l\right) d_{q, w} l\right]=c\left(z_{1}\right)\left[\int_{a}^{a^{\prime}} \zeta(l) d_{q, w} l-\left(a^{\prime}-a\right) z_{1}\right]=0 .
\end{aligned}
$$

\subsection{Hardy Inequalities with general kernels}

Theorem 3.2. Let

$$
\mathrm{I}_{1}=\left[\mathrm{a}, \mathrm{a}^{\prime}\right] \text { and } \mathrm{I}_{2}=\left[\mathrm{b}, \mathrm{b}^{\prime}\right] \quad \mathrm{a}, \mathrm{a}^{\prime}, \mathrm{b}, \mathrm{b}^{\prime} \in \mathbb{R}
$$

be two intervals. Define

$$
\mathrm{A}_{\varpi} \zeta\left(z_{1}\right)=\frac{1}{\bar{W}\left(z_{1}\right)} \int_{\mathrm{b}}^{\mathrm{b}^{\prime}} \varpi\left(z_{1}, z_{2}\right) \zeta\left(z_{2}\right) \mathrm{d}_{\mathbf{q}, w} z_{2} \quad z_{1} \in \mathrm{I}_{1}
$$

where $\varpi: I_{1} \times I_{2} \rightarrow \mathbb{R}$, is such that

$$
\bar{W}\left(z_{1}\right)=\int_{\mathrm{b}}^{\mathrm{b}^{\prime}} \varpi\left(z_{1}, z_{2}\right) \mathrm{d}_{\mathrm{q}, w}<\infty, \quad z_{1} \in \mathrm{I}_{1} .
$$

Also, $\eta: \mathrm{I}_{1} \rightarrow \mathbb{R}$ is such that

$$
\omega\left(z_{2}\right)=\int_{a}^{a^{\prime}} \frac{\varpi\left(z_{1}, z_{2}\right) \eta\left(z_{1}\right)}{\bar{W}\left(z_{1}\right)} \mathrm{d}_{\mathrm{q}, w} z_{1}<\infty, z_{2} \in \mathrm{I}_{2} .
$$

If $\Upsilon \in \mathrm{C}\left(\mathrm{I}_{1}, \mathbb{R}\right)$ is superquadratic where $\mathrm{I}_{1} \subset \mathbb{R}$ is an interval, then

$$
\begin{aligned}
& \int_{\mathbf{a}}^{\mathbf{a}^{\prime}} \Upsilon\left(A_{\varpi} \zeta\left(z_{1}\right)\right) \eta\left(z_{1}\right) \mathrm{d}_{\mathbf{q}, w}\left(z_{1}\right)+\int_{\mathbf{b}}^{\mathbf{b}^{\prime}} \int_{\mathbf{a}}^{\mathbf{a}^{\prime}} \Upsilon\left(\left|\zeta\left(z_{2}\right)-A_{\varpi} \zeta\left(z_{1}\right)\right|\right) \frac{\eta\left(z_{1}\right) \bar{W}\left(z_{1}\right)}{\bar{W}\left(z_{1}\right)} d_{\mathbf{q}, w}\left(z_{1}\right) d_{\mathbf{q}, w}\left(z_{2}\right) \\
& \quad \leqslant \int_{b}^{\mathbf{b}^{\prime}} \Upsilon\left(\zeta\left(z_{2}\right)\right) w\left(z_{2}\right) \mathrm{d}_{\mathbf{q}, w}\left(z_{2}\right)
\end{aligned}
$$

holds for all $\mathrm{q}, w$-integrable $\zeta: \mathrm{I}_{2} \rightarrow \mathbb{R}$ such that $\zeta\left(\mathrm{I}_{2}\right) \subset \mathrm{I}_{1}$.

Proof. Theorem 3.1 gives

$$
\begin{aligned}
\int_{a}^{a^{\prime}} \Upsilon\left(A_{\varpi} \zeta\left(z_{1}\right)\right) \eta\left(z_{1}\right) d_{\mathbf{q}, w}\left(z_{1}\right)= & \int_{a}^{a^{\prime}} \eta\left(z_{1}\right) \Upsilon\left(\frac{1}{\bar{W}\left(z_{1}\right)} \int_{b}^{b^{\prime}} \varpi\left(z_{1}, z_{2}\right) \zeta\left(z_{2}\right) d_{\mathbf{q}, w}\left(z_{2}\right)\right) d_{\mathbf{q}, w}\left(z_{1}\right) \\
\leqslant & \int_{a}^{a^{\prime}} \frac{\eta\left(z_{1}\right)}{\bar{W}\left(z_{1}\right)}\left(\int_{b}^{b^{\prime}} \varpi\left(z_{1}, z_{2}\right) \Upsilon\left(\zeta\left(z_{2}\right)\right) d_{\mathbf{q}, w}\left(z_{2}\right)\right) d_{\mathbf{q}, w}\left(z_{1}\right) \\
& -\int_{a}^{a^{\prime}} \frac{\eta\left(z_{1}\right)}{\bar{W}\left(z_{1}\right)} \int_{b}^{b^{\prime}} \varpi\left(z_{1}, z_{2}\right) \Upsilon\left(\left|\zeta\left(z_{2}\right)-A_{\varpi} \zeta\left(z_{1}\right)\right|\right) d_{\mathbf{q}, w}\left(z_{2}\right) d_{\mathbf{q}, w}\left(z_{1}\right) .
\end{aligned}
$$

By using (2.3), it can be written as

$$
\begin{aligned}
& =\int_{a}^{a^{\prime}} \frac{\eta\left(z_{1}\right)}{\bar{W}\left(z_{1}\right)}\left(\int_{w_{0}}^{b^{\prime}} \varpi\left(z_{1}, z_{2}\right) \Upsilon\left(\zeta\left(z_{2}\right)\right) d_{\mathbf{q}, w}\left(z_{2}\right)-\int_{\mathcal{w}_{\mathrm{o}}}^{b} \varpi\left(z_{1}, z_{2}\right) \Upsilon\left(\zeta\left(z_{2}\right)\right) d_{\mathbf{q}, w}\left(z_{2}\right)\right) \mathrm{d}_{\mathbf{q}, w}\left(z_{1}\right) \\
& \quad-\int_{a}^{a^{\prime}} \frac{\eta\left(z_{1}\right)}{\bar{W}\left(z_{1}\right)}\left(\int_{w_{\mathrm{o}}}^{b^{\prime}} \varpi\left(z_{1}, z_{2}\right) \Upsilon\left(\left|\zeta\left(z_{2}\right)-A_{\varpi} \zeta\left(z_{1}\right)\right|\right) d_{\mathbf{q}, w}\left(z_{2}\right)\right.
\end{aligned}
$$




$$
\begin{aligned}
& \left.-\int_{w_{0}}^{b} \varpi\left(z_{1}, z_{2}\right) \curlyvee\left(\left|\zeta\left(z_{2}\right)-A_{\varpi} \zeta\left(z_{1}\right)\right|\right) d_{q, w}\left(z_{2}\right)\right) d_{q, w}\left(z_{1}\right) \\
& =\int_{w_{\mathrm{o}}}^{\mathbf{a}^{\prime}} \frac{\eta\left(z_{1}\right)}{\bar{W}\left(z_{1}\right)}\left(\int_{\mathcal{w}_{\mathrm{o}}}^{\mathrm{b}^{\prime}} \varpi\left(z_{1}, z_{2}\right) \curlyvee\left(\zeta\left(z_{2}\right)\right) \mathrm{d}_{\mathbf{q}, w}\left(z_{2}\right)-\int_{\mathcal{w}_{\mathrm{o}}}^{\mathrm{b}} \varpi\left(z_{1}, z_{2}\right) \curlyvee\left(\zeta\left(z_{2}\right)\right) \mathrm{d}_{\mathbf{q}, w}\left(z_{2}\right)\right) \mathrm{d}_{\mathbf{q}, w}\left(z_{1}\right) \\
& -\int_{w_{\mathrm{o}}}^{\mathrm{a}} \frac{\eta\left(z_{1}\right)}{\bar{W}\left(z_{1}\right)}\left(\int_{w_{\mathrm{o}}}^{\mathrm{b}^{\prime}} \varpi\left(z_{1}, z_{2}\right) \Upsilon\left(\zeta\left(z_{2}\right)\right) \mathrm{d}_{\mathrm{q}, w}\left(z_{2}\right)-\int_{w_{\mathrm{o}}}^{\mathrm{b}} \varpi\left(z_{1}, z_{2}\right) \curlyvee\left(\zeta\left(z_{2}\right)\right) \mathrm{d}_{\mathrm{q}, w}\left(z_{2}\right)\right) \mathrm{d}_{\mathrm{q}, w}\left(z_{1}\right) \\
& -\int_{w_{\mathrm{o}}}^{\boldsymbol{a}^{\prime}} \frac{\eta\left(z_{1}\right)}{\bar{W}\left(z_{1}\right)}\left(\int_{w_{\mathrm{o}}}^{\mathbf{b}^{\prime}} \varpi\left(z_{1}, z_{2}\right) \Upsilon\left(\left|\zeta\left(z_{2}\right)-A_{\varpi} \zeta\left(z_{1}\right)\right|\right) \mathrm{d}_{\mathrm{q}, w}\left(z_{2}\right)\right. \\
& \left.-\int_{w_{\mathrm{o}}}^{\mathrm{b}} \varpi\left(z_{1}, z_{2}\right) \Upsilon\left(\left|\zeta\left(z_{2}\right)-A_{\varpi} \zeta\left(z_{1}\right)\right|\right) \mathrm{d}_{\mathrm{q}, w}\left(z_{2}\right)\right) \mathrm{d}_{\mathrm{q}, w}\left(z_{1}\right) \\
& -\int_{w_{\mathrm{o}}}^{\mathrm{a}} \frac{\eta\left(z_{1}\right)}{\bar{W}\left(z_{1}\right)}\left(\int_{w_{\mathrm{o}}}^{\mathrm{b}^{\prime}} \varpi\left(z_{1}, z_{2}\right) \Upsilon\left(\left|\zeta\left(z_{2}\right)-A_{\varpi} \zeta\left(z_{1}\right)\right|\right) \mathrm{d}_{\mathrm{q}, w}\left(z_{2}\right)\right. \\
& \left.-\int_{\mathcal{w}_{\mathrm{o}}}^{\mathrm{b}} \varpi\left(z_{1}, z_{2}\right) \Upsilon\left(\left|\zeta\left(z_{2}\right)-A_{\varpi} \zeta\left(z_{1}\right)\right|\right) \mathrm{d}_{\mathbf{q}, w}\left(z_{2}\right)\right) \mathrm{d}_{\mathbf{q}, w}\left(z_{1}\right) \\
& =a^{\prime} \sum_{m=0}^{\infty} q^{m} \frac{\eta\left(a_{m}^{\prime}\right)}{\bar{W}\left(a_{m}^{\prime}\right)}\left(b^{\prime} \sum_{n=0}^{\infty} q^{n} \varpi\left(a_{m}^{\prime}, b_{n}^{\prime}\right) \curlyvee\left(\zeta\left(b_{n}^{\prime}\right)\right)-b \sum_{n=0}^{\infty} q^{n} \varpi\left(a_{m}^{\prime}, b_{n}\right) \curlyvee\left(\zeta\left(b_{n}\right)\right)\right) \\
& -a \sum_{m=0}^{\infty} q^{m} \frac{\eta\left(a_{m}\right)}{\bar{W}\left(a_{m}\right)}\left(b^{\prime} \sum_{n=0}^{\infty} q^{n} \varpi\left(a_{m}, b_{n}^{\prime}\right) \Upsilon\left(\zeta\left(b_{n}^{\prime}\right)\right) b \sum_{n=0}^{\infty} q^{n} \varpi\left(a_{m}, b_{n}\right) \Upsilon\left(f\left(b_{n}\right)\right)\right) \\
& -a^{\prime} \sum_{m=0}^{\infty} q^{m} \frac{\eta\left(a_{m}^{\prime}\right)}{\bar{W}\left(a^{\prime}{ }_{m}\right)}\left(b^{\prime} \sum_{n=0}^{\infty} q^{n} \varpi\left(a_{m}^{\prime}, b_{n}^{\prime}\right) \Upsilon\left(\left|\zeta\left(b_{n}^{\prime}\right)-A_{\varpi}\left(a_{m}^{\prime}\right)\right|\right)\right. \\
& \left.-b \sum_{n=0}^{\infty} q^{n} \varpi\left(a_{m}^{\prime}, b_{n}\right) \Upsilon\left(\left|\zeta\left(b_{n}\right)-A_{\varpi}\left(a_{m}^{\prime}\right)\right|\right)\right) \\
& -a \sum_{m=0}^{\infty} q^{m} \frac{\eta\left(a_{m}\right)}{\bar{W}\left(a_{m}\right)}\left(b^{\prime} \sum_{n=0}^{\infty} q^{n} \varpi\left(a_{m}, b_{n}^{\prime}\right) \Upsilon\left(\left|\zeta\left(b_{n}^{\prime}\right)-A_{\varpi}\left(a_{m}\right)\right|\right)\right. \\
& \left.-b \sum_{n=0}^{\infty} q^{n} \varpi\left(a_{m}, b_{n}\right) \Upsilon\left(\left|\zeta\left(b_{n}\right)-A_{\varpi}\left(a_{m}\right)\right|\right)\right) \text {. }
\end{aligned}
$$

Switch the sums to get

$$
\begin{aligned}
& =b^{\prime} \sum_{n=0}^{\infty} q^{n}\left(\frac{a^{\prime} \sum_{m=0}^{\infty} q^{m} \varpi\left(a^{\prime}{ }_{m}, b^{\prime}{ }_{n}\right) \eta\left(a^{\prime}{ }_{m}\right)}{\bar{W}\left(a^{\prime}{ }_{m}\right)}-\frac{a \sum_{m=0}^{\infty} q^{m} \varpi\left(a_{m}, b^{\prime}{ }_{n}\right) \eta\left(a_{m}\right)}{\bar{W}\left(a_{m}\right)}\right) \curlyvee\left(\zeta\left(b^{\prime}{ }_{n}\right)\right) \\
& -b \sum_{n=0}^{\infty} q^{n}\left(\frac{a^{\prime} \sum_{m=0}^{\infty} q^{m} \varpi\left(a^{\prime}{ }_{m}, b_{n}\right) \eta\left(a^{\prime}{ }_{m}\right)}{\bar{W}\left(a^{\prime}{ }_{m}\right)}-\frac{a \sum_{m=0}^{\infty} q^{m} \varpi\left(a_{m}, b_{n}\right) \eta\left(a_{m}\right)}{\bar{W}\left(a_{m}\right)}\right) \curlyvee\left(\zeta\left(b_{n}\right)\right) \\
& -b^{\prime} \sum_{n=0}^{\infty} q^{n}\left(\frac{a^{\prime} \sum_{m=0}^{\infty} q^{m} \eta\left(a^{\prime}{ }_{m}\right) \varpi\left(a^{\prime}{ }_{m} b^{\prime}{ }_{n}\right) \Upsilon\left(\left|\zeta\left(b^{\prime}{ }_{n}\right)-A_{k} \zeta\left(a^{\prime}{ }_{m}\right)\right|\right)}{\bar{W}\left(a^{\prime}{ }_{m}\right)}\right. \\
& \left.-\frac{a \sum_{m=0}^{\infty} q^{m} \eta\left(a_{m}\right) \varpi\left(a_{m}, b^{\prime}{ }_{n}\right) \Upsilon\left(\left|\zeta\left(b^{\prime}{ }_{n}\right)-A_{k} \zeta\left(a_{m}\right)\right|\right)}{\bar{W}\left(a_{n}\right)}\right)
\end{aligned}
$$




$$
\begin{aligned}
& -b \sum_{n=0}^{\infty} q^{n}\left(\frac{a^{\prime} \sum_{m=0}^{\infty} q^{m} \eta\left(a_{m}^{\prime}\right) \varpi\left(a_{m}^{\prime}, b_{n}\right) \Upsilon\left(\left|\zeta\left(b_{n}\right)-A_{k} \zeta\left(a_{m}^{\prime}\right)\right|\right)}{\bar{W}\left(a_{m}^{\prime}\right)}\right. \\
& \left.-\frac{a \sum_{m=0}^{\infty} q^{m} \eta\left(a_{m}\right) \varpi\left(a_{m}, b_{n}\right) \Upsilon\left(\left|\zeta\left(b_{n}\right)-A_{k} \zeta\left(a_{m}\right)\right|\right)}{\bar{W}\left(a_{n}\right)}\right) \\
= & \int_{b}^{b^{\prime}} \Upsilon\left(\zeta\left(z_{2}\right)\right)\left(\int_{a}^{a^{\prime}} \frac{\varpi\left(z_{1}, z_{2}\right) \eta\left(z_{1}\right)}{\bar{W}\left(z_{1}\right)}\right) d_{q, w}\left(z_{2}\right) \\
& -\int_{b}^{b^{\prime}} \int_{a}^{a^{\prime}} \Upsilon\left(\left|\zeta\left(z_{2}\right)-A_{\varpi} \zeta\left(z_{1}\right)\right|\right) \frac{\eta\left(z_{1}\right) \varpi\left(z_{1}, z_{2}\right)}{\bar{W}\left(z_{1}\right)} d_{q, w}\left(z_{1}\right) d_{q, w}\left(z_{2}\right) \\
= & \int_{b}^{b^{\prime}} \Upsilon\left(\zeta\left(z_{2}\right)\right) w\left(z_{2}\right) d_{q, w}\left(z_{2}\right)-\int_{b}^{b^{\prime}} \int_{a}^{a^{\prime}} \Upsilon\left(\left|\zeta\left(z_{2}\right)-A_{\varpi} \zeta\left(z_{1}\right)\right| \frac{\eta\left(z_{1}\right) \varpi\left(z_{1}, z_{2}\right)}{\bar{W}\left(z_{1}\right)} d_{q, w}\left(z_{1}\right) d_{q, w}\left(z_{2}\right),\right.
\end{aligned}
$$

which gives (3.7).

Remark 3.3. If the function $\Upsilon$ is subquadratic, then the Jensen's inequality for superquadratic function is reversed, which means that reversed sign in (3.7) holds.

Corollary 3.4. Assume (3.3)-(3.6) hold. If $\mathrm{p} \geqslant 2$, then

$$
\begin{aligned}
\int_{a}^{a^{\prime}} & A_{\varpi}^{p} \zeta\left(z_{1}\right) \eta\left(z_{1}\right) d_{\mathbf{q}, w}\left(z_{1}\right)+\int_{b}^{\mathbf{b}^{\prime}} \int_{a}^{a^{\prime}}\left|\zeta\left(z_{1}\right)-A_{\varpi} \zeta\left(z_{1}\right)\right|^{p} \frac{\eta\left(z_{1}\right) \varpi\left(z_{1}, z_{2}\right)}{\bar{W}\left(z_{1}\right)} \mathrm{d}_{\mathbf{q}, w}\left(z_{1}\right) \mathrm{d}_{\mathbf{q}, w}\left(z_{2}\right) \\
& \leqslant \int_{b}^{\mathbf{b}^{\prime}} \zeta^{p}\left(z_{2}\right) w\left(z_{2}\right) \mathrm{d}_{\mathbf{q}, w}\left(z_{2}\right)
\end{aligned}
$$

holds for all $\mathrm{q}, w$ integrable $\mathrm{g}_{1}: \mathrm{I}_{2} \rightarrow \mathbb{R}_{+}$. If $0<\mathrm{p} \leqslant 2$, then above inequality holds in reversed direction.

Proof. Put $\Upsilon\left(z_{1}\right)=z_{1}$ p in Theorem 3.2.

Remark 3.5. In particular, if, $p=2$ in Corollary 3.4, the following identity is obtained

$$
\begin{aligned}
& \int_{\mathbf{a}}^{\mathbf{a}^{\prime}} A_{\varpi}^{2} \zeta(\zeta) \eta\left(z_{1}\right) \mathrm{d}_{\mathrm{q}, w}\left(z_{1}\right)+\int_{\mathrm{b}}^{\mathrm{b}^{\prime}} \int_{\mathbf{a}}^{\mathbf{a}^{\prime}}\left|\zeta\left(z_{1}\right)-A_{\varpi} \zeta\left(z_{1}\right)\right|^{2} \frac{\eta\left(z_{1}\right) \varpi\left(z_{1}, z_{2}\right)}{\bar{W}\left(z_{1}\right)} \mathrm{d}_{\mathbf{q}, w}\left(z_{1}\right) \mathrm{d}_{\mathbf{q}, w}\left(z_{2}\right) \\
& \quad \leqslant \int_{\mathbf{b}}^{\mathbf{b}^{\prime}} \zeta^{2}\left(z_{2}\right) w\left(z_{2}\right) \mathrm{d}_{\mathbf{q}, w}\left(z_{2}\right) .
\end{aligned}
$$

Corollary 3.6. Assume (3.6)-(3.7) hold. If $\mathrm{P} \geqslant 1$, then

$$
\int_{\mathbf{a}}^{\mathbf{a}^{\prime}} \eta\left(z_{1}\right) \exp \left(A_{\varpi} g(x)\right) d_{\mathbf{q}, w}(a)+I \leqslant \int_{b}^{\mathbf{b}^{\prime}} g^{\mathfrak{p}}\left(z_{2}\right) w\left(z_{2}\right) d_{\mathbf{q}, w}\left(z_{2}\right)
$$

holds for all $\mathrm{q}, w$ integrable $\mathrm{g}: \mathrm{I}_{2} \rightarrow(0, \infty)$ with

$$
A_{\varpi} g\left(z_{1}\right):=\frac{P}{\bar{W}\left(z_{1}\right)} \int_{b}^{b^{\prime}} \varpi\left(z_{1}, z_{2}\right) \ln g\left(z_{2}\right) d_{q, w}\left(z_{2}\right)
$$

and

$$
I=\int_{b}^{b^{\prime}} \int_{a}^{a^{\prime}} e^{\left|\ln g\left(z_{2}\right)-A_{\varpi} f\left(z_{1}\right)\right|^{p} \frac{\eta\left(z_{1}\right) \varpi\left(z_{1}, z_{2}\right)}{W\left(z_{1}\right)}} d_{q, w}\left(z_{1}\right) d_{q, w}\left(z_{2}\right) .
$$

Proof. Use $\Upsilon\left(z_{1}\right)=e^{z_{1}}-z_{1}-1$ and $f=\ln \left(g^{p}\right)$ in Theorem 3.2. 
Corollary 3.7. Assume (3.6)-(3.7) hold, then

$$
\int_{a}^{a^{\prime}} \eta\left(z_{1}\right) \exp \left(A_{\varpi} g\left(z_{1}\right)\right) d_{q, w}\left(z_{1}\right)+I \leqslant \int_{b}^{b^{\prime}} g\left(z_{2}\right) w\left(z_{2}\right) d_{q, w}\left(z_{2}\right)
$$

holds for all $\mathrm{q}, w$ integrable $\mathrm{g}: \mathrm{I}_{2} \rightarrow(0, \infty)$ with

$$
A_{\varpi} g\left(z_{1}\right):=\frac{1}{\bar{W}\left(z_{1}\right)} \int_{\mathrm{b}}^{\mathrm{b}^{\prime}} \varpi\left(z_{1}, z_{2}\right) \ln \mathrm{g}\left(z_{2}\right) \mathrm{d}_{\mathrm{q}, w}\left(z_{2}\right)
$$

and

$$
I=\int_{b}^{b^{\prime}} \int_{a}^{a^{\prime}} e^{\left|\operatorname{lng} g\left(z_{2}\right)-A_{\varpi} \zeta\left(z_{1}\right)\right| \frac{\eta\left(z_{1}\right) \varpi\left(z_{1}, z_{2}\right)}{W\left(z_{1}\right)}} d_{q, w}\left(z_{1}\right) d_{q, w}\left(z_{2}\right) .
$$

Proof. Use $p=1$ in Corollary 3.6.

Corollary 3.8. Let (3.3)-(3.7) hold and denote $\int_{a}^{a^{\prime}} d_{q, \omega} z_{2}=\left|a^{\prime}-a\right|, \int_{b}^{b^{\prime}} d_{q, \omega} z_{2}=\left|b^{\prime}-b\right|$, such that $\mid a^{\prime}-$ $\mathrm{a}|,| \mathrm{b}^{\prime}-\mathrm{b} \mid<\infty$. If $\checkmark \in \mathrm{C}(\mathrm{I}, \mathrm{R})$ is superquadratic, then

$$
\begin{aligned}
& \int_{a}^{a^{\prime}} \Upsilon\left(\frac{1}{\left|b^{\prime}-b\right|} \int_{b}^{b^{\prime}} \zeta\left(z_{2}\right) d_{q, \omega} z_{2}\right) d_{q, \omega} z_{1} \\
& \quad+\frac{1}{\left|b^{\prime}-b\right|} \int_{b}^{b^{\prime}} \int_{a}^{a^{\prime}} \Upsilon\left(\zeta\left(z_{2}\right)-\frac{1}{\left|b^{\prime}-b\right|} \int_{b}^{b^{\prime}} \zeta\left(z_{2}\right) d_{q, \omega}\right) d_{q, \omega} z_{1} d_{q, \omega} z_{2} \leqslant \frac{a^{\prime}-a}{b^{\prime}-b} \int_{b}^{b^{\prime}} \Upsilon\left(\zeta\left(z_{2}\right)\right) d_{q, \omega} z_{2}
\end{aligned}
$$

holds for all d, w-integrable $\zeta: \mathrm{I}_{2} \rightarrow \mathrm{R}$ such that $\zeta\left(\mathrm{I}_{2}\right) \subset \mathrm{I}_{1}$. If function $\Upsilon$ is subquadratic, then above inequality is reversed.

Proof. It follows form Theorem 3.2 by taking $\varpi\left(z_{1}, z_{2}\right)=1$ and $\eta\left(z_{1}\right)=1$, in this case $\bar{W}(b)=\int_{b}^{b^{\prime}} d_{q, \omega} z_{2}=$ $\left|\mathrm{b}^{\prime}-\mathrm{b}\right|$ and $\omega z_{2}=\int_{\mathrm{a}}^{\mathrm{a}^{\prime}} \frac{1}{\left|\mathrm{a}^{\prime}-\mathrm{a}\right|} \mathrm{d}_{\mathrm{q}, \omega} z_{1}=\frac{\left|\mathrm{a}^{\prime}-\mathrm{a}\right|}{\mathrm{b}^{\prime}-\mathrm{b}}$.

3.3. Hardy inequalities with special kernels

In this section we assume

$$
\mathrm{I}=\mathrm{I}_{1}=\mathrm{I}_{2}=\left[\mathrm{a}, \mathrm{a}^{\prime}\right), \quad 0 \leqslant \mathrm{a}<\mathrm{a}^{\prime} \leqslant \infty .
$$

Theorem 3.9. Suppose $\eta: \mathrm{I} \rightarrow \mathbb{R}_{+}$is a $\mathrm{q}$, w-integrable function and denote

$$
\tilde{\omega}\left(z_{2}\right)=\int_{z_{2}}^{a^{\prime}} \frac{\eta\left(z_{1}\right)}{\left(\left(q z_{1}+\omega\right)-a\right)} d_{q, \omega} z_{1} .
$$

If $\Upsilon \in \mathrm{C}(\mathrm{I}, \mathrm{R}), \mathrm{I} \subset \mathbb{R}$, is superquadratic function, then

$$
\begin{aligned}
& \int_{a}^{a^{\prime}} \eta\left(z_{1}\right) \Upsilon\left(\tilde{A_{\varpi}} \zeta\left(z_{1}\right)\right) d_{q, \omega} z_{1}+\int_{a}^{a^{\prime}} \int_{z_{2}}^{a^{\prime}} \frac{\eta\left(z_{1}\right)}{\left(\left(q z_{1}+\omega\right)-a\right)} \Upsilon\left(\zeta\left(z_{2}\right)-\tilde{A_{\varpi}} \zeta\left(z_{1}\right)\right) d_{q, \omega} z_{1} d_{q, \omega} z_{2} \\
& \quad \leqslant \int_{a}^{a^{\prime}} \tilde{\omega}\left(z_{2}\right) \Upsilon\left(\zeta\left(z_{2}\right)\right) d_{q, \omega} z_{2}
\end{aligned}
$$

holds for all q, $\omega$-integrable $\zeta: \mathrm{I} \rightarrow \mathbb{R}$ such that $\zeta(\mathrm{I}) \subset \mathrm{I}$, where

$$
\left(\left(\tilde{A}_{\varpi}\right) \zeta\right)\left(z_{1}\right)=\frac{1}{\left(\left(\mathbf{q} z_{1}+\omega\right)-\mathbf{a}\right)} \int_{\mathbf{a}}^{\left(\mathbf{q} z_{1}+\omega\right)} \zeta\left(z_{2}\right) \mathrm{d}_{\mathbf{q}, \omega} z_{2} .
$$

If $\Upsilon$ is subquadratic, then (3.9) is reversed. 
Proof. Statement follows from Theorem 3.2 by using

$$
\varpi\left(z_{1}, z_{2}\right)=\left\{\begin{array}{ll}
1, & \text { if } a \leqslant z_{2}<\mathrm{q} z_{1}+\omega \leqslant \mathbf{a}^{\prime} \\
0, & \text { otherwise, }
\end{array}\right\}
$$

since in this case

$$
\bar{W}\left(z_{1}\right)=\int_{a}^{q x+\omega} d_{q, \omega} z_{2}=\left(\left(q z_{1}+\omega\right)-a\right)
$$

and thus $A_{\varpi}=\tilde{A_{\varpi}}, \omega=\tilde{\omega}$.

Corollary 3.10. In (3.8) if we take $\mathrm{a}=0$ and $\Upsilon \in \mathrm{C}(\mathrm{I}, \mathrm{R})$ is superquadratic for $\mathrm{I} \subset \mathbb{R}$, then

$$
\begin{aligned}
& \int_{0}^{a^{\prime}} \Upsilon\left(A_{\varpi} \zeta\left(z_{1}\right)\right) \frac{d_{q, \omega} z_{1}}{z_{1}}+\int_{0}^{a^{\prime}} \int_{z_{2}}^{a^{\prime}} \Upsilon\left(\left|\zeta\left(z_{2}\right)-A_{\varpi} \zeta\left(z_{1}\right)\right|\right) \frac{d_{q, \omega} z_{1}}{z_{1}\left(q z_{1}+\omega\right)} d_{q, \omega} z_{2} \\
& \quad \leqslant \int_{0}^{a^{\prime}} \omega\left(z_{2}\right) \Upsilon\left(\zeta\left(z_{2}\right)\right) d_{\mathbf{q}, \omega} z_{2}
\end{aligned}
$$

holds for all $\mathrm{q}, \omega$-integrable $\zeta: \mathrm{I} \rightarrow \mathbb{R}$ such that $\zeta(\mathrm{I}) \subset \mathrm{I}$, where

$$
\left(A_{\varpi} \zeta\right)\left(z_{1}\right)=\frac{1}{\left(\mathbf{q} z_{1}+\omega\right)} \int_{0}^{q z_{1}+\omega} \zeta\left(z_{2}\right) \mathrm{d}_{\mathbf{q}, \omega} z_{2} .
$$

Proof. Use $\eta\left(z_{1}\right)=\frac{1}{z_{1}}$ in Theorem 3.9, since in this case

$$
\omega\left(z_{2}\right)=\int_{z_{2}}^{a^{\prime}} \frac{1}{z_{1}\left(\mathbf{q} z_{1}+\omega\right)} d_{\mathbf{q}, \omega} z_{1}=\left(\frac{1}{z_{2}}-\frac{1}{a^{\prime}}\right) .
$$

Remark 3.11. If $b=\infty$, then inequality (3.10) takes the form

$$
\int_{0}^{\infty} \Upsilon\left(A_{\varpi} \zeta\left(z_{1}\right)\right) \frac{d_{\mathrm{q}, \omega} z_{1}}{z_{1}}+\int_{0}^{\infty} \int_{z_{2}}^{\infty} \Upsilon\left(\left|\zeta\left(z_{2}\right)-A_{\varpi} \zeta\left(z_{1}\right)\right|\right) \frac{d_{\mathrm{q}, \omega} z_{1}}{z_{1}\left(\mathrm{q} z_{1}+\omega\right)} d_{\mathrm{q}, \omega} z_{2} \leqslant \int_{0}^{\infty} \Upsilon\left(\zeta\left(z_{2}\right)\right) \frac{\mathrm{d}_{\mathrm{q}, \omega} z_{2}}{z_{2}} .
$$

If $\Upsilon$ is subquadratic then above inequality is reversed.

Theorem 3.12. Let us take $\mathrm{a} \geqslant 0, \mathrm{a}^{\prime}=\infty$ in (3.8) and let $\varphi: I \rightarrow \mathbb{R}_{+}$be defined by

$$
\varphi\left(z_{2}\right)=\frac{1}{\left(\mathbf{q} z_{2}+\omega\right)} \int_{\mathbf{a}}^{\mathrm{q} z_{2}+\omega} \mathrm{d}_{\mathbf{q}, \omega} z_{1}=\left(1-\frac{\mathrm{a}}{\left(\mathbf{q} z_{2}+\omega\right)}\right) .
$$

If $\Upsilon \in \mathrm{C}(\mathrm{I}, \mathbb{R})$ is superquadratic, then

$$
\begin{aligned}
& \int_{\mathbf{a}}^{\infty} \Upsilon\left(z_{1} \int_{z_{1}}^{\infty} \frac{\zeta\left(z_{2}\right)}{z_{2}\left(\mathrm{q} z_{2}+\omega\right)} \mathrm{d}_{\mathrm{q}, \omega} z_{2}\right) \frac{\mathrm{d}_{\mathrm{q}, \omega} z_{1}}{z_{1}} \\
& +\int_{a}^{\infty} \int_{a}^{\mathrm{q} z_{2}+\omega} \Upsilon\left(\left|\zeta\left(z_{2}\right)-z_{1} \int_{z_{1}}^{\infty} \frac{\zeta\left(z_{2}\right)}{z_{2}\left(\mathrm{q} z_{2}+\omega\right)} \mathrm{d}_{\mathrm{q}, \omega} z_{2}\right|\right) \mathrm{d}_{\mathrm{q}, \omega} z_{1} \frac{\mathrm{d}_{\mathrm{q}, \omega} z_{2}}{z_{2}\left(\mathrm{q} z_{2}+\omega\right)} \leqslant \int_{\mathbf{a}}^{\infty} \varphi\left(z_{2}\right) \Upsilon\left(z_{2}\right) \frac{\mathrm{d}_{\mathrm{q}, \omega} z_{2}}{z_{2}}
\end{aligned}
$$

holds for all q, w-integrable $\zeta: \mathrm{I} \rightarrow \mathbb{R}$ such that $\zeta(\mathrm{I}) \subset \mathrm{I}$. If $\Upsilon$ is subquadratic, then above inequality is reversed.

Proof. Statement follows from Theorem 3.9, by using

$$
\varpi\left(z_{1}, z_{2}\right)=\left\{\begin{array}{ll}
\frac{1}{z_{2}\left(q z_{2}+\omega\right)}, & \text { for } z_{2} \geqslant z_{1}, \\
0, & \text { otherwise, }
\end{array}\right\}
$$

since in this case $\bar{W}\left(z_{1}\right)=\frac{1}{z_{1}}$, and replace $\eta\left(z_{1}\right)$ with $\frac{1}{z_{1}}$, to obtain $\omega\left(z_{2}\right)=\frac{\varphi\left(z_{2}\right)}{z_{2}}$.

Remark 3.13. Let $\Upsilon(v)=v^{p}$ in Theorems 3.9 and 3.12, then for $p \geqslant 2$ the corresponding inequalities are reversed. However, for $p \in(0,2]$ the corresponding inequalities will be preserved. 


\subsection{Polya-Knopp type inequalities}

Corollary 3.14. Assume the hypothesis (3.8) with $\mathrm{a} \geqslant 0$ and $\mathrm{a}^{\prime}=\infty$. If $\Upsilon \in \mathrm{C}(\mathrm{I}, \mathrm{R})$ is superquadratic, then

$$
\begin{aligned}
& \int_{a}^{\infty} \eta\left(z_{1}\right) \Upsilon\left(\frac{1}{\left(\mathbf{q} z_{1}+\omega\right)-a} \int_{a}^{\left(\mathbf{q} z_{1}+\omega\right)} \zeta\left(z_{2}\right) d_{\mathbf{q}, \omega} z_{2}\right) d_{\mathbf{q}, \omega} z_{1}+\int_{a}^{\infty} \int_{z_{2}}^{\infty} \frac{\eta\left(z_{1}\right)}{\left(\left(\mathbf{q} z_{1}+\omega\right)-\mathbf{a}\right)} \\
& \times \Upsilon\left(\mid \zeta\left(z_{2}\right)-\left(\left\{\frac{1}{\left(\mathbf{q} z_{1}+\omega\right)-\mathrm{a}} \int_{\mathbf{a}}^{\left(\mathbf{q} z_{1}+\omega\right)} \zeta\left(z_{2}\right) \mathrm{d}_{\mathbf{q}, \omega} z_{2} \mid\right)\right) \mathrm{d}_{\mathbf{q}, \omega} z_{1} \mathrm{~d}_{\mathbf{q}, \omega} z_{2}\right. \\
& \leqslant \int_{a}^{\infty} \tilde{\omega}\left(z_{2}\right) \Upsilon\left(\zeta\left(z_{2}\right)\right) d_{\mathrm{q}, \omega} z_{2}
\end{aligned}
$$

holds for all $\mathrm{q}$, w-integrable $\mathrm{g}_{1}: \mathrm{I} \rightarrow \mathbb{R}$.

Proof. The statement follows from Theorem 3.9.

Example 3.15. Use $\Upsilon=z_{1}{ }^{p}, p>1$ in (3.11), to get the inequality

$$
\begin{aligned}
& \int_{a}^{\infty} \eta\left(z_{1}\right)\left(\frac{1}{\left(\mathbf{q} z_{1}+\omega\right)-a} \int_{a}^{\left(q z_{1}+\omega\right)} \zeta\left(z_{2}\right) \tilde{d}_{\mathbf{q}, \omega} z_{2}\right)^{p} \mathrm{~d}_{\mathbf{q}, \omega} z_{1} \\
& +\int_{a}^{\infty} \int_{z_{2}}^{\infty} \frac{\eta\left(z_{1}\right)}{\left(\left(\mathbf{q} z_{1}+\omega\right)-a\right)}\left(\mid \zeta\left(z_{2}\right)-\left(\frac{1}{\left(q z_{1}+\omega\right)-a} \int_{a}^{\left(\mathbf{q} z_{1}+\omega\right)} \zeta\left(z_{2}\right) d_{\mathbf{q}, \omega} z_{2} \mid\right)\right)^{p} d_{q, \omega} z_{1} d_{q, \omega} z_{2} \\
& \leqslant \int_{a}^{\infty} \tilde{\omega}\left(z_{2}\right)\left(\zeta\left(z_{2}\right)\right)^{p} d_{q, \omega} z_{2} \text {. }
\end{aligned}
$$

Example 3.16. Use $\Upsilon=e^{z_{1}}-z_{1}-1$ and $\zeta\left(z_{1}\right)=\ln g\left(z_{1}\right)$ for $p \geqslant 1$ in (3.11), to get

$$
\begin{aligned}
& \int_{\mathbf{a}}^{\infty} \eta\left(z_{1}\right) e^{\frac{1}{\left(\mathrm{q} z_{1}+\omega\right)-\mathrm{a}} \int_{\mathrm{a}}^{\left(\mathrm{q} z_{1}+\omega\right)} \zeta\left(z_{2}\right) \mathrm{d}_{\mathrm{q}, \omega} z_{2}} \mathrm{~d}_{\mathrm{q}, \omega} z_{1} \\
& \quad+\int_{\mathbf{a}}^{\infty} \int_{z_{2}}^{\infty} \frac{\eta\left(z_{1}\right)}{\left(\left(\mathrm{q} z_{1}+\omega\right)-\mathrm{a}\right)} e^{\left|\zeta\left(z_{2}\right)-\left(\frac{1}{\left(\mathrm{q} z_{1}+\omega\right)-\mathrm{a}} \int_{\mathrm{a}}^{\left(\mathrm{q} z_{1}+\omega\right)} \zeta\left(z_{2}\right) \mathrm{d}_{\mathrm{q}, \omega} z_{2}\right)\right|} d_{\mathrm{q}, \omega} z_{1} \mathrm{~d}_{\mathrm{q}, \omega} z_{2} \\
& \quad \leqslant \int_{a}^{\infty} \tilde{\omega}\left(z_{2}\right)\left(\zeta\left(z_{2}\right)\right)^{\mathrm{p}} \mathrm{d}_{\mathrm{q}, \omega} z_{2} .
\end{aligned}
$$

Remark 3.17. If superquadratic function reduces to convex function, then we get results given in [4].

\section{References}

[1] S. Abramovich, G. Jameson, G. Sinnamon, Refining Jensen's inequality, Bull. Math. Soc. Sci. Math. Roumanie, 47 (2004), 3-14. 2.1

[2] S. Abramovich, K. Krulić, J. Pečarić, L.-E. Persson, Some new refined Hardy type inequalities with general kernel and measures, Aequat. Math., 79 (2010), 157-172. 1.2

[3] M. H. Annaby, A. E. Hamza, K. A. Aldwoah, Hahn Difference Operator and Associated Jackson-Nörlund Integrals, J. Optim. Theory Appl., 154 (2012), 133-153. 1.1, 2.3

[4] N. Ashraf, K. A. Khan, S. Mobeen, A. Nosheen, Inequalities of Hardy type for Jackson Nörlund integrals, Commun. Math. Appli., 9 (2018), 411-419. 1.2, 3.17

[5] G. Bangerezako, Variational calculus on q-nonuniform lattices, J. Math. Anal. Appl., 306 (2005), 161-179. 1.1

[6] J. Barić, A. Nosheen, J. Pečarić, Time scale Hardy-type inequalities with general kernel for superquadratic functions, Proc. A. Razmadze Math. Inst., 165 (2014), 13-30. 1.2

[7] R. Bibi, A. Nosheen, J. Pečarić, Refinements of Hardy-type inequalities on time scales, Commun. Math. Anal., 17 (2014), 67-87. 1.2

[8] T. Donchev, A. Nosheen, J. Pečarić, Hardy-type inequalities on time scales via convexity in several variables, ISRN Math. Anal., 2013 (2013), 9 pages. 1.2

[9] W. Hahn, Über orthogonalpolynome, die q-differenzenlgleichungen genü gen, Math. Nachr., 2 (1949), 4-34. 1.1

[10] G. H. Hardy, J. E. Littlewood, G. Pólya, Inequalities, Cambrige University Press, (1959). 1.2 
[11] K. A. Khan, A. Nosheen, J. Pečarić, n-Exponential convexity of some dynamic Hardy-type functionals, J. Math. Inequal., 8 (2014), 331-347. 1.2

[12] A. Kufner, L. Maligranda, L.-E. Persson, The prehistory of the Hardy Inequality, Amer. Math. Monthly, 113 (2006), 715-732.

[13] A. Kufner, L. Maligranda, L.-E. Persson, The Hardy Inequality: About its history and some related results, Vydavatelsky Servis Publishing House, Pilsen, (2007).

[14] A. Kufner, L.-E. Persson, Weighted inequalities of Hardy type, World Scientific Publishing Co, New Jersey, (2003). 1.2

[15] A. B. Malinowska, N. Martins, Generalized transversality condition for the Hahn quantum variational calculus, Optimization, 62 (2011), 323-344. 1.1

[16] A. B. Malinowska, D. F. M. Torres, The Hahn quantum variational calculus, J. Optim. Theory Appl., 147 (2010), 419-442. 1.1 